\title{
Novel Hypoglycemic Injury Mechanism: NMDA Receptor-Mediated White Matter Damage
}

Running Head: NMDA receptors mediate WM hypoglycemic injury

Yang $\mathrm{X}^{1}$, Hamner $\mathrm{MA}^{2}$, Brown $\mathrm{AM}^{3}$, Evans $\mathrm{R}^{3}$, Ye $\mathrm{Z}^{2}$, Chen $\mathrm{SD}^{1}$ and Ransom $\mathrm{BR}^{2 *}$

${ }^{1}$ Department of Neurology

Ruijin Hospital affiliated to Shanghai Jiaotong University School of Medicine, Shanghai 200025, China

2Department of Neurology

University of Washington

Seattle, WA 98195

${ }^{3}$ School of Biomedical Sciences

University of Nottingham

Nottingham, NG2 5HY, UK

${ }^{*}$ Corresponding author:

Bruce R. Ransom, MD, PhD

Department of Neurology 
University of Washington, Rm RR650

1959 NE Pacific Street

Seattle, WA 98195

Email: bransom@uw.edu

Office: $206-543-2340$

Number of characters in title: 79

Number of characters in Running Head: 40

Number of words in Abstract: 245

Number of Words in body of manuscript: 7532

Number of Figures: 8

Number of Color Figures: 1

Number of Tables: 0

Acknowledgment:

This work was supported by funding from the NIH/NINDS (R01

NS015589; B.R.R.), National Natural Science Foundation of China (NSFC 30900453 and 81070958; X.Y.) and the University of Nottingham (A.M.B.). 


\section{ABSTRACT}

Objective: Hypoglycemia is a common adverse event and can injure central nervous system (CNS) white matter (WM). We determined if glutamate receptors were involved in hypoglycemic WM injury.

Methods: Mouse optic nerves (MON), CNS WM tracts, were maintained at $37^{\circ} \mathrm{C}$ with oxygenated artificial cerebrospinal fluid (ACSF) containing $10 \mathrm{mM}$ glucose. Aglycemia was produced by switching to 0 glucose ACSF. Supra-maximal compound action potentials (CAPs) were elicited using suction electrodes and axon function was quantified as the area under the CAP. Amino acid release was measured using HPLC. Extracellular [lactate] was measured using an enzyme electrode.

Results: About $50 \%$ of MON axons were injured after 60 min of aglycemia (90\% after $90 \mathrm{~min}$ ); injury was not affected by animal age. Blockade of NMDA-type glutamate receptors improved recovery after $90 \mathrm{~min}$ of aglycemia by $250 \%$. Aglycemic injury was increased by reducing $\left[\mathrm{Mg}^{2+}\right]_{0}$ or increasing [glycine $]_{0}$, and decreased by lowering $\mathrm{pH}_{0}$, expected results for NMDA receptor-mediated injury. Extracellular $\mathrm{pH}$ increased during aglycemia, due to a drop in [lactate-]o. Aglycemic injury was dramatically reduced in the absence of $\left[\mathrm{Ca}^{2+}\right]_{0}$. Extracellular aspartate, a selective NMDA receptor agonist, increased during aglycemia.

Interpretation: Aglycemia injured WM by a unique excitotoxic mechanism involving NMDA receptors (located primarily on oligodendrocytes). During WM aglycemia, the selective NMDA agonist, aspartate, is released, probably from astrocytes. Injury is mediated by $\mathrm{Ca}^{2+}$ influx through aspartate-activated NMDA receptors made permeable by an accompanying alkaline shift in $\mathrm{pH}_{0}$ caused by a fall in [lactate $]_{0}$. These insights have important clinical implications. 


\section{INTRODUCTION}

Hypoglycemia continues to be an important and common adverse clinical event in patients with diabetes, and more rarely with other conditions ${ }^{1-4}$. The central nervous system (CNS) is extremely vulnerable to dysfunction and injury with hypoglycemia ${ }^{1,5,6}$. In addition, there is compelling evidence that CNS dysfunction can develop slowly over years in patients with well controlled diabetes, presumably related to subclinical episodes of hypoglycemia ${ }^{7,8}$. For these reasons, it is important to understand how hypoglycemia produces irreversible CNS injury. This knowledge will provide a basis for development of effective therapies to minimize the extent of damage and improve clinical outcome.

Recent observations have drawn attention to selective white matter (WM) damage as a consequence of protracted hypoglycemia 9, 10; in fact, WM can be the predominant site of injury 9,10 . However, experimental studies on the mechanisms of hypoglycemic injury have used primarily rodents. As discussed elsewhere, humans have roughly 4 to 5 -fold more WM than rodents, a critical difference because WM and gray matter (GM) are vastly different tissues with unique mechanisms of injury ${ }^{11,12}$.

Fortunately, validated and practical models of CNS WM injury are now available ${ }^{13-16}$. Initial studies using the acutely isolated mouse optic nerve (MON), a myelinated WM tract, found that 60 minutes of aglycemia produced substantial irreversible injury. The mechanism of injury was found to be $\mathrm{Ca}^{2+-d e p e n d e n t ~ a n d ~}$ to involve activation of L-type $\mathrm{Ca}^{2+}$ channels and reverse $\mathrm{Na}^{+} / \mathrm{Ca}^{2+}$ exchange ${ }^{17}$. These two pathways mediated toxic $\mathrm{Ca}^{2+}$ influx contributing to permanent loss of axonal excitability ${ }^{17}$. Subsequently, another form of energy disruption in WM, ischemia, was found to produce injury mediated by excessive activation of excitatory glutamate receptors. This pathogenesis, called excitotoxicity, was first defined in CNS gray matter (GM) ${ }^{11}$. The characteristics of 'excitotoxicity' in WM are distinct from GM; in other words, the toxic glutamate receptor activation seen in WM during ischemia is a 'special form' of excitotoxicity $11,16,18-20$. While both $\mathrm{N}$ - 
methyl-D-aspartate (NMDA) and non-NMDA receptors have been found in WM, only non-NMDA receptors appear to participate in ischemic (i.e., oxygen and glucose deprivation (OGD)) WM injury 14, 18, 20-22, but see ${ }^{23}$ ). This was somewhat unexpected because NMDA receptors are clearly expressed by myelinating oligodendrocytes 18,24 and these cells are unquestionably injured during energy deprivation $18,19,21,22,25$.

We sought to determine if excitotoxicity might also be involved in the WM injury seen with aglycemia. Indeed, we found that excitotoxicity contributed importantly to this special type of irreversible WM injury but unlike the situation with OGD, in aglycemia NMDA receptors were prominently involved, in a $\mathrm{Ca}^{2+}$ dependent manner. Our results also provided a plausible explanation for why NMDA receptors were involved in aglycemia but not in ischemia. Finally, we showed that WM aglycemic injury arose from NMDA receptors activated by aspartate, not glutamate. These findings raise important questions about how to optimally manage the clinical situation of severe hypoglycemic brain dysfunction. 


\section{METHODS AND MATERIALS}

All experiments were done in accordance with the University of Washington Institutional Animal Care and Use Committee.

\section{Electrophysiology}

Mouse optic nerves (MONs) were acutely obtained from C57BL/6 mice that varied in age from one to 24 months of age; most experiments were done on three month-old mice. As previously described ${ }^{26}$, mice were deeply anesthetized with $\mathrm{CO}_{2}$ and then decapitated. Optic nerves were dissected free and cut at the optic chiasm and behind the orbit. The optic nerves were freed from their dural sheaths and placed in an interface perfusion chamber (Medical Systems Corp., Greenvale, NY, USA) and maintained at $37^{\circ} \mathrm{C}$. MONs were superfused with artificial cerebrospinal fluid (ACSF) containing (in mmol/L): $125 \mathrm{NaCl}, 3.0 \mathrm{KCl}$, $2.0 \mathrm{CaCl}_{2}, 2.0 \mathrm{MgSO}_{4} \cdot 7 \mathrm{H}_{2} \mathrm{O}, 1.25 \mathrm{NaH}_{2} \mathrm{PO}_{4}, 26 \mathrm{NaHCO}_{3}$, and 10 glucose. The ACSF was bubbled with an $\mathrm{O}_{2}$-free gas mixture $\left(95 \% \mathrm{~N}_{2}: 5 \% \mathrm{CO}_{2}\right)$ to maintain $\mathrm{pH}$ at 7.45. A humidified gas mixture of $95 \% \mathrm{O}_{2} / 5 \% \mathrm{CO}_{2}$ continuously aerated the chamber. Two sets of suction electrodes were placed in the bath to allow recording from two optic nerves at the same time. Suction electrodes back-filled with the appropriate ACSF were used for stimulating and recording. The stimulating electrode was attached to the rostral end of the nerve, while the proximal end was attached to a second electrode to record the compound action potential (CAP), ensuring orthodromic stimulation. Stimulus pulse (30 $\mu \mathrm{s}$ duration) strength (Isostim 520; WPI, Sarasota, FL) was adjusted to evoke the maximum CAP and then increased another $25 \%$ (i.e., supramaximal stimulation $)^{13}$. During an experiment, the supramaximal CAP was elicited every 30 seconds. The recording electrode was connected to an amplifier (Standford Research Systems, Model SR 560) and the signal was amplified 500 times, filtered at $30 \mathrm{kHz}$ and acquired at $20 \mathrm{kHz}$.

Nerves were allowed to equilibrate for at least 30 mins before recording commenced. In glucose deprivation experiments, the solution in the stimulating 
and recording electrodes was switched to glucose-free ACSF (i.e., aglycemia). Osmotic compensation was achieved by adding $10 \mathrm{mmol} / \mathrm{L}$ sucrose. $\mathrm{Ca}^{2+}$ free ACSF was made by omitting $\mathrm{CaCl}_{2}$ and adding $0.5 \mathrm{mmol} / \mathrm{L}$ ethylene glycol-bis (Baminoethylether)-N,N,N',N'-tetraacetic acid (EGTA) with equimolar $\mathrm{MgCl}_{2}$. Zero $\mathrm{Mg}^{2+} \mathrm{ACSF}$ was made by omitting $\mathrm{MgSO}_{4}$ and replacing with equimolar $\mathrm{Na}_{2} \mathrm{SO}_{4}$. In a few experiments, both oxygen and glucose were deleted to create an ischemia-like condition, also referred to as oxygen-glucose deprivation or OGD.

Bath $\mathrm{pH}$ was changed from 7.4 to 7.0 by reducing the $\left[\mathrm{HCO}_{3}^{-}\right]$using the following ACSF recipe (in mmol/L): $141 \mathrm{NaCl}, 3.0 \mathrm{KCl}, 2.0 \mathrm{CaCl}_{2}, 2.0 \mathrm{MgSO}_{4}$ $.7 \mathrm{H}_{2} \mathrm{O}, 1.25 \mathrm{NaH}_{2} \mathrm{PO}_{4}, 10 \mathrm{NaHCO}_{3}$ and 10 glucose. All changes in ACSF were introduced 15 mins before the insult and continued until 15 mins after terminating the insult (e.g., $\left.{ }^{18}\right)$.

Pharmacological agents were applied for 15 mins before the insult and continued until 15 mins after terminating the insult.. The following agents were purchased from Tocris (Ellisville, Missouri): 2,3-Dioxo-6-nitro-1,2,3,4tetrahydrobenzo[f]quinoxaline-7-sulfonamide (NBQX) (30 $\mu \mathrm{M}$; AMPA/Kainate channel blocker; dissolved in DMSO as $30 \mathrm{mM}$ stock solution), 7-chlorokynurenic acid (7-CKA) (50 $\mu \mathrm{M}$; glycine binding site blocker; dissolved in DMSO as $50 \mathrm{mM}$ stock solution) and (5S,10R)-(+)-5-Methyl-10,11-dihydro $\square-5 \mathrm{H}$ dibenzo[a, d]cyclohepte n-5,10-imine maleate (MK801) (15 $\mu \mathrm{M}$; NMDA receptor blocker; dissolved in DMSO as $15 \mathrm{mM}$ stock solution).

\section{Lactate biosensor}

Lactate and null biosensors were purchased from Sarissa Biomedical Ltd (Coventry, UK). In these experiments, however, the lactate signals were sufficiently large that subtraction of the null signal did not meaningfully alter the lactate signal amplitude. The lactate biosensors ( $25 \mu \mathrm{m} \square$ in diameter and $500 \mu \mathrm{m}$ in length) were pressed against the pial-glial membrane of the MON.

Experimental recordings began after an equilibration period of 30 to 60 minutes. At the beginning and end of all experiments, lactate biosensors were calibrated 
using lactate concentrations of 10,100 and $1000 \square \mathrm{M}$. Results were considered valid only if the pre- and post-calibrations deviated by no more than $5 \%$.

\section{pH-sensitive microelectrodes}

Ion-sensitive microelectrodes for extracellular $\mathrm{pH}\left(\mathrm{pH}_{0}\right)$ measurements were made according to the method of Borrelli et al., ${ }^{27}$ with slight modifications ${ }^{6}$. Briefly, double-barrel microelectrodes were pulled and beveled to a tip diameter of 2 to $5 \mu \mathrm{m}$. The ion-sensitive barrel was back-filled with a short column of $\mathrm{H}^{+-}$ sensitive sensor (Fluka pH ionophore). The indifferent barrel was back-filled with $140 \mathrm{mmole} / \mathrm{L} \mathrm{NaCl}+20 \mathrm{mmol} / \mathrm{L}$ HEPES adjusted to $\mathrm{pH}$ 7.0. Only electrodes that showed near-Nernstian responses to 10 fold changes to $\left[\mathrm{H}^{+}\right]$calibrating solutions were used in experiments.

\section{Data analysis}

Optic nerve function was quantitatively determined by integrating the area under the CAP. Data were acquired online (Digidata 1440A; Molecular Devices, Sunnyvale, CA) using proprietary software (Clampex, Molecular Devices). CAP area was calculated using pClamp (Molecular Devices) and was normalized by averaging the baseline CAP area over a period of 15 minutes, and setting this value to 1.0. The normalized CAP area at any time is proportional to the relative number of functioning axons ${ }^{13}$. Data are presented as the mean, and standard error of the mean (SEM), of normalized traces. Statistical significance was determined by unpaired, two-tailed Student's t-test. $P$ values less than 0.05 were considered significant.

\section{Glutamate, aspartate and glycine measurements}

Amino acid (i.e., glutamate, aspartate, glycine, etc.) release from MON was determined by first collecting the superfusate during 60 or $90 \mathrm{~min}$ aglycemia (or OGD in a few experiments) and then subjecting these samples to quantitative amino acid measurement using HPLC ${ }^{28}$. Experiments were designed to monitor amino acid release simultaneously from 1 pair of MONs (this technique can be 
modified to enhance sensitivity by using 5 pairs of MONs measured at one time). Briefly, amino acids were pre-column derivatized with o-phthaldialdehyde (Sigma, St Louis, MO), separated and measured using standard techniques. Samples of extracellular perfusion fluid were collected continuously such that every vial contained two min of superfusate. Glutamate content was measured in every other vial (i.e., every 4 mins), except during the baseline when it was measured every 2 mins. Collected samples were centrifuged at $16,000 \mathrm{~g}$ for 3 mins and supernatants transferred for HPLC analysis. Glutamate measurements, normalized to baseline glutamate release were made from MONs treated identically to those studied electrophysiologically and the results were plotted against time. The rate and release pattern of glutamate was monitored for $30 \mathrm{~min}$ before injury (60 mins and 90 mins aglycemia or 60 mins OGD), and was continued for at least 30 min after the end of the insult. Actual amounts of glutamate measured in $\mathrm{mM}$ were determined by comparing the experimental measurements to standard samples.

Intracellular amino acid content of cells within the MON was determined using a slightly modified established technique ${ }^{29}$. Briefly, pairs of MONs were sonicated in $0.2 \mathrm{ml}$ of homogenization solution (distilled $\mathrm{H}_{2} \mathrm{O}$ supplemented with $0.4 \mathrm{mM}$ DTT and $1 \mathrm{mM}$ EDTA). The homogenate was diluted 10 -fold in homogenization solution prior to protein measurement and HPLC measurement of amino acids. Aliquots for HPLC measurement of amino acids were then acidified by $\mathrm{HCl}$ and centrifuged. The supernatant was harvested and neutralized by $\mathrm{NaOH}$ prior to using HPLC to measure amino acid concentrations. Protein content was measured in identical aliquots using Bio-Rad protein assay reagent. 


\section{$\underline{\text { RESULTS }}$}

\section{Aglycemia caused duration-dependent WM injury}

As previously described ${ }^{6,30}$, aglycemia caused loss of WM excitability, measured as failure of the CAP. The pattern of CAP loss with aglycemia was unique because the CAP was largely unchanged for the first 10 to $15 \mathrm{~min}$ following glucose deprivation (Fig. 1A, B) ${ }^{30}$. Once the CAP began to fail, all three peaks fell in unison (data not shown). When glucose was restored after aglycemia, the CAP partially recovered to a stable new level (e.g., Fig. 1A). The duration of aglycemia dictated the extent of CAP recovery (Fig. 1B). The area under the CAP, which is proportional to the number of excited axons (see ${ }^{13}$ ), recovered to $72.2 \pm 6.3 \%, 48.2 \pm 3.7 \%$ or $5.0 \pm 1.2 \%$ of control CAP area after 30,60 or 90 min of aglycemia, respectively. The extent of CAP recovery following a given period of aglycemia stabilized after about $30 \mathrm{~min}$ and remained stable during observation periods as long as 4 hours. For quantification and comparison, the area of CAP recovery was determined 60 min following the conclusion of glucose deprivation ${ }^{30}$.

\section{Glycogen content affected the extent of aglycemic injury}

It is known that tissue glycogen content affects the latency to CAP failure after removing glucose; more glycogen prolonged and less glycogen shortened latency to CAP failure ${ }^{31}$. The present study focused on CAP recovery after standard periods of aglycemia and the role of glycogen must be considered. As shown in Figure 1C, the extent of recovery was significantly less if glycogen was first depleted by pre-incubation for one hour in $2 \mathrm{mM}$ glucose, rather than the 'normal' glucose of $10 \mathrm{mM}^{31}$. With this important variable in mind, all subsequent experiments were done after one-hour incubation in $10 \mathrm{mM}$ glucose to ensure a consistent level of glycogen.

\section{Aglycemic WM injury did not depend on animal age}


The extent of WM injury due to ischemia is dependent on animal age; for a given period of ischemia, tissue from older animals shows significantly greater injury ${ }^{18}$. Similar experiments were done to test for age-dependency of hypoglycemic WM injury. The extent of injury after $60 \mathrm{~min}$ of aglycemia, measured as CAP recovery, was determined in four groups of animals ranging in age from 1 month to 24 months. The extent of irreversible injury was similar for all age groups indicating that age did not affect aglycemia-induced injury extent (Fig. 1D), as is the case with ischemia ${ }^{18}$.

\section{Aglycemic injury was mediated by NMDA receptors}

The mechanism(s) underlying aglycemic WM injury were investigated.

AMPA/kainate-type glutamate receptors participate in ischemic WM injury 14, 22, 28 and we tested for involvement of these receptors in aglycemic injury. The dosage of NBQX that was maximally protective for ischemic WM injury had no significant effect on CAP recovery after 60 min of aglycemia (control vs. NBQX CAP: $49.2 \pm$ $3.7 \%$ vs. $46.2 \pm 5.8 \%, p>0.05$ ), and only weakly improved CAP recovery after 90 min of aglycemia (Fig. $2 A$; control vs. NBQX CAP recovery: $5.0 \pm 1.2 \%$ vs. $12.2 \pm 1.8 \%, p<0.01)$.

Blocking NMDA-type glutamate receptors does not protect against ischemic WM injury ${ }^{18,32}$, in fact it significantly worsens this injury ${ }^{18}$. The NMDA antagonist MK801 was tested and, surprisingly, this agent powerfully improved CAP recovery after both 60 (see data summary below )and 90 min of aglycemia (Fig. $2 A ; 30.8 \pm 5.5 \%, p<0.0005$ ). In addition to dramatically improving CAP recovery, MK801 delayed the onset of CAP decline (Fig. 2A). A second NMDA antagonist, 7-CKA, was applied in the same manner as MK801 and similarly improved CAP recovery after aglycemia (Fig. $2 \mathrm{~B}$; control recovery $=6.4 \% \pm 1.3 \%$ vs. 7 -CKA recovery $=34.9 \pm 4.1 \%, p<0.001)$.

It was possible that the high dose of NBQX used in these experiments, 30 $\mu \mathrm{M}$, might have weakly, and non-specifically, blocked NMDA receptors ${ }^{33}$. If so, no additional benefit would be expected if NBQX were added to MK801. We found exactly that result (Fig. 2C). The amount of CAP recovery seen after 90 
min of aglycemia was greatly improved by MK801, compared to control, but CAP recovery did not improve beyond this level when NBQX was added (Fig. 2C). These results strongly argue that NMDA receptors were the principal, probably the only, type of glutamate receptor responsible for irreversible CAP loss following aglycemia.

NMDA-type glutamate receptors (NMDAR) are modulated in a highly characteristic manner by several factors, including extracellular $\left[\mathrm{Mg}^{2+}\right]\left(\left[\mathrm{Mg}^{2+}\right]_{0}\right)$, extracellular [glycine] and extracellular $\mathrm{pH}\left(\mathrm{pH}_{0}\right)^{34}$. Physiological [ $\left.\mathrm{Mg}^{2+}\right]_{0}$ blocks the NMDAR pore at normal membrane potential. Reducing $\left[\mathrm{Mg}^{2+}\right]_{0}$ by application of $\mathrm{Mg}^{2+}-$ free ACSF would be expected to overcome this block and facilitate pore opening and ion flux upon NMDAR activation. The effect of $\mathrm{Mg}^{2+-}$-free ACSF on aglycemia-induced WM injury was determined (Fig. 3A). In the presence of greatly reduced $\left[\mathrm{Mg}^{2+}\right]_{0}, 60 \mathrm{~min}$ of aglycemia was much more damaging; CAP recovery decreased from $48.05 \pm 5.3 \%$ to $14.9 \pm 4.4 \%\left(p<4.1 \times 10^{-5}\right)$. The worsened outcome with application of $\mathrm{Mg}^{2+}$-free ACSF was completely reversed by MK801 (applied with $0 \mathrm{Mg}^{2+}$ ), indicating that this effect depended entirely on NMDAR activation (Fig. 3A).

Reduced $\left[\mathrm{Mg}^{2+}\right]_{0}$, however, had no significant effect on the extent of CAP recovery after $60 \mathrm{~min}$ of ischemia (ischemia recovery $=22.1 \pm 4.7 \% \mathrm{vs}$. ischemia $+0 \mathrm{Mg}^{2+}$ recovery $=28.2 \pm 5.5 \%, \mathrm{p}=0.43$; data not shown), consistent with the fact that NMDARs are not involved in the pathophysiology of WM ischemic injury 18. This result eliminated concern about a non-specific effect of reducing $\left[\mathrm{Mg}^{2+}\right]_{\mathrm{o}}$.

The NMDAR is unique in requiring two agonists for activation, glutamate and glycine (or d-serine) ${ }^{34}$. We reasoned that glycine availability might limit the extent of NMDAR activation during aglycemia, and tested this possibility by providing glycine (1 mM) in ACSF during aglycemia (Fig. 3B). Increasing glycine exogenously during aglycemia led to significantly less recovery $(36.51 \pm 7.00 \%$ vs. $48.19 \pm 3.70 \%, p<0.03)$. This result supported the idea that NMDARs were 
involved in aglycemia-induced WM injury and that [glycine] was 'rate limiting' in terms of the extent of NMDAR activation.

\section{Extracellular lactate fell rapidly during aglycemia accompanied by an alkaline shift}

Extracellular lactate was measured directly in the MON using a commercially available 'enzyme' electrode specific for lactate (see methods; ${ }^{35}$ ). The size of the electrode necessitated measurement at the pial-glial boundary of the nerve as shown diagrammatically in Figure 4A. In the presence of normal bath glucose (i.e., $10 \mathrm{mM}$ ), extracellular [lactate-] ([lactate- $]_{0}$ ) was zero in the bath and averaged $0.46 \pm 0.02 \mathrm{mM}(n=6)$ when the electrode was pressed against the MON. This value reflected [lactate] $]_{0}$ within the optic nerve but will generally be less than the 'true' nerve [lactate-]o because of an unknown amount of bath dilution. The [lactate $]_{0}$ in the nerve was stable over time and did not change when bath glucose was lowered to $5 \mathrm{mM}$ or increased to $20 \mathrm{mM}$ (data not shown; see discussion).

During aglycemia, [lactate-]o declined rapidly after about $5 \mathrm{~min}$ and fell to near zero in about $15 \mathrm{~min}$ (Fig. 4B). Extracellular $\mathrm{pH}\left(\mathrm{pH}_{\circ}\right)$ within the optic nerve was measured using $\mathrm{pH}$-sensitive microelectrodes placed toward the center of the nerve. As previously reported, $\mathrm{pH}_{\circ}$ was about $0.2 \mathrm{pH}$ units more acid than the bath $\mathrm{pH}(\sim 7.4)^{6,36}$. The fall in [lactate-]o during aglycemia was accompanied by a temporally related increase in $\mathrm{pH}_{0}$ which ultimately plateaued at about 7.4, near the bath $\mathrm{pH}$ (Fig. $4 \mathrm{C}$ ). The synchronous initiation of changes in [lactate- $]_{0}$ and $\mathrm{pH}_{0}$ occurred well before the CAP began to fall. When the CAP reached its minimum during aglycemia, an inflection point was often seen in the alkaline shift of $\mathrm{pH}_{\circ}$ (arrow) suggesting that there were two stages to the alkaline shift (Fig 4C; see discussion). After switching back to normal bath glucose, [lactate- $]_{0}$ and $\mathrm{pH}_{\circ}$ rapidly returned to their original values, although [lactate- $]_{0}$ transiently overshot its baseline level. 
The proton-binding site on the NMDAR renders it exquisitely sensitive to $\mathrm{pH}_{\mathrm{o}}$ over the normal physiological range ${ }^{37}$. Acidic shifts in $\mathrm{pH}_{\circ}$ block, while alkaline shifts facilitate, NMDAR-mediated ion fluxes. Aglycemia caused an alkaline shift in $\mathrm{pH}_{\mathrm{o}}$, due to the fall in [lactate-]o, which would enhance NMDARmediated ion fluxes and, presumably, injury. This idea was tested by altering $\mathrm{pH}_{\circ}$ during aglycemia (Fig. 4D; see methods). The aglycemia-induced injury was compared in bath solutions identical except for $\mathrm{pH}_{\circ}$ (7.45 vs 7.20; Fig. 4D). CAP recovery after 90 min of aglycemia was significantly greater in the more acidic bath solution, as predicted if proton-sensitive NMDARs are a primary step in the pathogenesis of aglycemic WM injury. Interestingly, the latencies to the start of CAP decline and to complete CAP loss were both markedly delayed in the acidic solution.

\section{Aglycemic WM injury was dependent on extracellular $\left[\mathrm{Ca}^{2+}\right]$}

An important feature of NMDARs is high permeability to $\mathrm{Ca}^{2+}$ and this characteristic can lead to neural injury. If NMDARs are involved in mediating aglycemic WM injury, a high degree of dependence on extracellular [ $\left.\mathrm{Ca}^{2+}\right]$ $\left(\left[\mathrm{Ca}^{2+}\right]_{0}\right)$ is expected. In experiments on three month-old MONs, the extent of CAP recovery after 90 min of aglycemia was enormously increased when the insult occurred in the absence of extracellular $\mathrm{Ca}^{2+}$ (from $4.97 \pm 1.2 \%$ to $86.93 \pm$ $3.6 \%, p<0.00001$; Fig. 5A). It was previously noted in older animals (i.e., $>6$ months of age) that exposure to $\mathrm{Ca}^{2+}$-free ACSF in conjunction with ischemia caused a delayed CAP deterioration following the insult ${ }^{18}$. This paradoxical result, still unexplained, was not seen in older animals exposed to aglycemia in the presence of $\mathrm{Ca}^{2+}$-free ACSF. In other words, $\mathrm{Ca}^{2+}$-free ACSF afforded dramatic and persistent protection against aglycemic WM injury regardless of age (Fig. 5B).

Experimental results from the above experiments are summarized in figure 6 . The results from 90 minutes and 60 minutes of aglycemia are shown in panels $A$ and $B$, respectively. Note that NBQX did not improve CAP recovery 
from 60 minutes of aglycemia (Fig. 6B). Although NBQX had a small protective effect against injury due to 90 minutes of aglycemia, this was not additive to the much greater MK801 protection. Taken together, these results indicated that AMPA/kainate receptors were not involved in mediating aglycemic injury, or at most had a minimal effect compared to NMDARs.

\section{Amino acid release during aglycemia}

Glutamate is robustly released during ischemia in WM and activates AMPA/Kainate receptors that mediate irreversible WM injury 18, 28. Aglycemic injury, on the other hand, occurred through activation of NMDARs with little or no involvement of AMPA/Kainate receptors (see above). Experiments to detect glutamate release during aglycemia were performed. Using HPLC, glutamate concentration was measured in sequential aliquots of bath solution passing over the optic nerve ${ }^{28}$. No apparent change in glutamate release was detected during $40 \mathrm{~min}$ of aglycemia (Fig. 8A). When aglycemia was switched to oxygen/glucose deprivation (i.e., ischemia), however, glutamate release increased about 10 -fold over about $35 \mathrm{~min}$, as previously reported (Fig 7A; ${ }^{28}$ ). Aspartate can also activate NMDARs and might be involved in hypoglycemic injury of gray matter areas like the hippocampus ${ }^{5}$. Like glutamate, no aspartate release could be detected during aglycemia but it was clearly released by a following episode of OGD (Fig 7B). Longer periods of aglycemia, up to $90 \mathrm{~min}$, also failed to demonstrate any obvious change in glutamate or aspartate release (not shown).

These results did not eliminate the possibility of subtle changes in glutamate or aspartate release during aglycemia. The standard method employed would not be able to detect very small changes in amino acid release into the extracellular space of the optic nerve because tissue extracellular volume is minute in comparison to bath volume, introducing a large dilution factor ${ }^{28}$. To increase the resolution for detecting small amounts of amino acid release, further experiments were done using ten MONs, rather than two (see methods). Because the volume of bath perfusion solution remained unchanged, or was 
slightly less because of displacement by the eight additional nerves, this strategy magnified the concentration of released amino acid by at least a factor of five. Using this modification, aspartate release was clearly detected during aglycemia (Fig 7D). It also became clear that glutamate release actually decreased significantly during aglycemia (Fig 7C). The concentrations of the NMDAR coagonists, glycine and d-serine, were also measured and their release was not significantly changed during aglycemia (data not shown).

A further analysis of amino acid changes during algycemia was carried out by measuring amino acid content of MON tissue under control conditions and after 30 min of aglycemia (Fig 7E). This provided a measure of intracellular amino acid content. During aglycemia, intracellular aspartate concentration increased about four-fold while intracellular glutamate concentration decreased by at least four-fold. Intracellular glycine concentration did not change significantly during aglycemia. These findings provide a rational explanation for the measured changes in the release of glutamate and aspartate during aglycemia (i.e., Figs 7C and 7D).

Taken together, these results indicated that aglycemia caused activation of NMDARs, very likely due to aspartate released into the extracellular space. Involvement of glutamate, the canonical agonist for NMDARs, seemed very unlikely because its intracellular concentration and extracellular release both fell during aglycemia. During ischemia, glutamate is released in a manner that is sensitive to blockade of $\mathrm{Na}^{+}$-dependent glutamate transport ${ }^{28}$. Aspartate is also a substrate for this transporter. When $\mathrm{Na}^{+}$-dependent glutamate/aspartate transport was blocked during algycemia by TBOA, CAP recovery was significantly increased (Fig 7F). This supported the idea that the NMDAR agonist released during aglycemia (i.e., aspartate) was likely released from an intracellular pool via reversal of the glutamate/aspartate $\mathrm{Na}^{+}$-dependent transporter. 


\section{DISCUSSION}

Hypoglycemia is a common cause of neurologic symptoms ${ }^{1-4,38}$. If severe and prolonged, hypoglycemia causes irreversible neural injury $\left(^{5}\right)$. Most studies on the mechanisms of hypoglycemic brain dysfunction and/or injury have focused on GM. This is unfortunate because WM also suffers from glucose deprivation and damage to this region contributes to clinical deficits ${ }^{9,10}$, which is not surprising in light of the fact that WM represents a major portion (about 55\%) of human forebrain volume ${ }^{39}$. Moreover, the mechanisms of WM injury are distinctive compared to GM ( ${ }^{40}$; see below). Finally, recent clinical reports show that WM can be selectively and severely injured by hypoglycemia ${ }^{9,10}$. Therapy for severe hypoglycemia, therefore, must benefit both WM and GM in order to be clinically effective.

We studied WM hypoglycemic injury and found that this injury was caused, in part, by excitotoxicity mediated by NMDARs, most likely activated by aspartate released into the extracellular space. This is surprising because a related insult, ischemia (i.e., oxygen/glucose deprivation or OGD), injures WM via AMPA/kainate receptors, and NMDARs are not involved ${ }^{18,22}$ (see below). Our conclusion is supported by the following observations: 1) hypoglycemic injury was significantly blocked by NMDAR antagonists (but minimally by AMPA/kainate receptor antagonists), 2) aspartate release was detected during aglycemia, and aspartate activates NMDARs but not other glutamate receptors, 3) glutamate release fell during aglycemia precluding activation of AMPA/ kainate receptors, 4) conditions known to favor activation of, and ion permeation through, NMDARs (reduced $\left[\mathrm{Mg}^{2+}\right]_{0}$ and increased [glycine] $]_{0}$ ) worsened injury, 5) during aglycemia $\mathrm{pH}_{\mathrm{o}}$ increased (i.e., proton concentration fell) due to a fall in [lactate-], relieving the 'proton block' that reduces ion fluxes through activated NMDARs, and 6) algycemic injury was greatly mitigated by removal of $\left[\mathrm{Ca}^{2+}\right]_{\mathrm{o}}$ as would be expected if injury depended on NMDAR-mediated $\mathrm{Ca}^{2+}$ influx. These findings have important implications for the management of severe hypoglycemia because WM may be among the first brain regions affected ${ }^{10}$. They suggest that early treatment with a suitable antagonist of NMDARs may be beneficial. 
Beyond this, they clarify the importance of extracellular ionic environment as a precondition for NMDAR involvement in neural injury.

\section{Amino Acid Release During Hypoglycemia}

One of the most striking differences between WM ischemia and WM aglycemia is the pattern of amino acid release induced by the two conditions. During ischemia, glutamate and aspartate are released into the extracellular space after a latency period of about $20 \mathrm{~min}\left({ }^{18,28}\right.$; Fig $\left.8 \mathrm{~A}, \mathrm{~B}\right)$. The latency is directly related to the time necessary for astrocyte glycogen to be depleted 28,30 . During aglycemia, in marked contrast, glutamate release into the extracellular space actually decreased (e.g., Fig. 7C). Given that ischemia and aglycemia both result in marked loss of tissue ATP ${ }^{41}$, and that this is associated with failure of extracellular glutamate homeostasis powered by ATP-dependent uptake, the absence of increased glutamate release into the extracellular space during aglycemia was unexpected.

The likely explanation is the well-known ability of brain tissue, including WM, to utilize substrates other than glucose for energy metabolism (e.g., ${ }^{42}$ ). While glucose is the primary energy substrate transported across the bloodbrain-barrier, brain cells themselves can use a variety of substrates as substitutes for glucose including fructose, glutamine and ketone bodies. Glutamate may also be used as a fuel ${ }^{42}$. Glutamate, glutamine and ketone bodies produce energy by entering the Krebs cycle, a metabolic sequence with an absolute dependence on the presence of $\mathrm{O}_{2}$. This fact, the necessity of $\mathrm{O}_{2}$, is the key for understanding why glutamate release is robust in ischemia, and absent in aglycemia (actually, the level of extracellular glutamate fell during aglycemia; Fig 7C). During aglycemia, glutamate and related metabolites are consumed in a desperate effort to maintain a normal level of ATP. Indeed, we found that intracellular [glutamate] fell to $25 \%$ of control level (Fig 7E). In ischemia, however, the Krebs cycle is paralyzed due to the absence of $\mathrm{O}_{2}$ and glutamate accumulates, available for export to the extracellular space (Fig. 7A) ${ }^{28}$. 
The final piece of this metabolic puzzle relates to aspartate and why it predominates during aglycemia. In the absence of glucose and the consequent glycolytic production of pyruvate, the Kreb's cycle intermediate, oxaloacetate, accumulates and lacks its normal condensation partner, acetyl-CoA (derived directly from pyruvate). Elevated intracellular oxaloacetate, in turn, drives aspartate production via the aspartate-glutamate transaminase reaction ${ }^{5,43}$. Our data indicated that there was roughly a four-fold increase of intracellular [aspartate] during aglycemia (Fig. 7E). Aspartate, as well as glutamate, is transported by $\mathrm{Na}^{+}$-dependent glutamate transporters. During aglycemia, this process can be expected to run in reverse and export aspartate to the extracellular space (i.e., Fig. 7D) ${ }^{44} 45$. Evidence for this sequence of events has been noted for more than a quarter century but all prior studies have been on GM areas of the brain ${ }^{44}$. Aspartate selectively activates NMDARs, and does so at low concentrations ${ }^{34}$.

\section{Model of Aglycemia-mediated Excitotoxic WM Injury}

The observations reported here indicate that aglycemia sets in motion a unique cascade of events producing irreversible CNS WM injury. Before presenting this model in detail, however, an important loose end must be addressed. The critical involvement of NMDARs in aglycemic WM injury begs the question of where these receptors are located. Experimental evidence indicates that oligodendrocytes are probably the main cell type in WM expressing NMDARs 18, 24, 46;Dr. Frank Kirchhoff, personal communication). The critical functional subunit of NMDARs, NR1, was expressed in rodent optic nerves of all ages ${ }^{18}$. A mouse has been created in which NR1 is selectively deleted in oligodendrocytes ( $F$. Kirchhoff, personal communication). Optic nerves from animals lacking oligodendrocyte NR1 showed a $60 \%$ reduction in total NR1 content. The residual NR1 expression appeared to be located on astrocytes and/or NG2 cells (F. Kirchhoff, personal communication). Based on these findings, we may conclude that NMDARs are heavily expressed on 
oligodendrocytes. Moreover, NMDA receptors on oligodendrocytes in the optic nerve are clearly functional and mediate $\mathrm{Ca}^{2+}$ influx when activated ${ }^{24,46}$. The exact distribution of NMDARs on oligodendrocytes from older animals remains unexplored but in young or developing cells of this lineage NMDARs are expressed primarily on processes and myelin 24,46 . The extent to which WM astrocytes or NG2 cells might express functional NMDARs in WM is simply not known at this time.

In normal WM, the supply of $\mathrm{O}_{2}$ and glucose is sufficient to maintain adequate levels of ATP (Fig 8A). The function of WM is safeguarded by the presence of glycogen in astrocytes which can serve as an emergency fuel supply during intense neural activity when fuel demand might outstrip supply ${ }^{31}$ or during short periods of glucose deprivation ${ }^{30}$. Extracellular glutamate is maintained at very low levels that are below the threshold for activation of AMPA/kainate receptors. Even if the high-affinity NMDARs on oligodendrocytes are partially occupied by glutamate under physiological conditions, they would not conduct ion fluxes without cell depolarization and relief of the proton block that exists at physiological $\mathrm{pH}^{34}$.

Before considering the events put in motion by aglycemia, it is instructive to look closely at the pathophysiology of WM injury due to ischemia, the essential condition of stroke (Fig 8B). This form of metabolic deprivation forms a natural counterpoint to the more selective metabolic disturbance caused by glucose deprivation. In fact, the confusion has existed that these conditions, which both reduce tissue ATP, might consequently damage cells in a similar fashion (e.g., ${ }^{47}$ ). This is most emphatically not the case in WM and the points of difference between the injurious steps unleashed by these two pathologies highlight remarkable differences of the upmost clinical importance. It is also germane to note that the quality of information about WM ischemic injury is detailed and robust, enjoying substantial cross validation by independent research groups (11, 12, 22). 
Ischemia causes energy metabolism to fail in all the component cells of WM, including axons, oligodendrocytes and astrocytes. A functional level of energy metabolism persists in astrocytes until glycogen in exhausted (\#1). In the absence of $\mathrm{O}_{2}$, lactate levels rise and cause an extracellular acidosis (\#2). A consequence of acidosis is 'proton block' of NMDARs. With exhaustion of ATP, $\left[\mathrm{Na}^{+}\right]_{i}$ increases (\#3) causing glutamate release into the extracellular space via reverse $\mathrm{Na}^{+}$-dependent glutamate transport (probably mediated by astrocytes)(\#4). In turn, glutamate activates AMPA/kainaite receptors on oligodendrocytes and their processes, including myelin (\#5), leading to intracellular ionic derangements and irreversible injury (\#6). The strong acidosis blocks ion fluxes in NMDA receptors, preventing them from participating in the injury cascade. Not shown in figure $8 \mathrm{~B}$ are the ionic derangements in axons that also participate in ischemic WM injury (e.g., ${ }^{48}$ )

With severe hypoglycemia, energy metabolism fails as astrocyte glycogen is exhausted (\#1). Because the Krebs cycle remains functional in the presence of $\mathrm{O}_{2}$, glutamate can be consumed as an alternative fuel, driving down both intracellular and extracellular [glutamate] (\#2). As discussed above, glutamate also interacts with accumulating oxaloacetate to form much higher concentrations of intracellular aspartate (\#3). As ATP falls, the $\mathrm{Na}^{+}$pump fails, $\left[\mathrm{Na}^{+}\right]_{i}$ increases and $\mathrm{Na}^{+}$-dependent glutamate/aspartate uptake reverses leading to increasing extracellular [aspartate] (\#4). Aspartate is an exclusive, and highaffinity, agonist for NMDARs and activates these receptors that are located primarily on oligodendrocyte processes and myelin. Both intracellular and extracellular [lactate] fall due to lactate consumption in the Krebs cycle after conversion to pyruvate (\#5). This is associated with an increase in $\mathrm{pH}_{0}$ causing relief of the 'proton' block of NMDARs and leading to toxic ion fluxes, especially $\mathrm{Ca}^{2+}$, that damage oligodendrocytes and myelin (\#6 and 7). The damage may be more focused on distal oligodendrocyte processes and myelin based on the greater density of NMDARs in these areas ${ }^{46}$. 


\section{Clinical Implications}

It is instructive to compare the mechanisms of hypoglycemic injury in WM and $\mathrm{GM}$, acknowledging at the outset that the pathophysiology of hypoglycemic injury is not completely understood. In part this is due to the fact that most prior experimental work on this topic has focused on $\mathrm{GM}^{5}$, 49. In $\mathrm{GM}$, hypoglycemia causes release of both glutamate and aspartate into the extracellular space. Glutamate released in GM probably comes from glutamate-containing synaptic vesicles because it is reduced by ablation of glutamatergic synaptic terminals ${ }^{49}$. Because glutamate, but not aspartate, activates both non-NMDA, as well as NMDA, glutamate receptors, it is immediately clear that the absence of extracellular glutamate release in WM during hypoglycemia constitutes a major difference in how GM and WM are injured by this deprivation. Antagonism of NMDA receptors is essential to minimize WM damage but blockade of both receptor subtypes appears to be important to protect $\mathrm{GM} 50$.

In summary, our results coupled with recent clinical studies showing that hypoglycemic encephalopathy is common in WM and may begin there ${ }^{9,10}$, suggest that patients presenting in hypoglycemic coma may benefit from treatment with an NMDAR antagonist. 


\section{FIGURES}

Figure 1.

Recovery of WM function after aglycemia depended on the duration of aglycemia and tissue glycogen, but not on age. (A) Shown is the effect of a standard $60 \mathrm{~min}$ period of aglycemia on normalized CAP area for a single mouse optic nerve (MON; 3-month-old animal; see Methods). Inset shows representative CAPs before (a), during (b) and after (c) aglycemia (CAPs elicited every $0.5 \mathrm{sec}$ ). Aglycemia caused CAP failure that recovered in normal glucose to $45 \%$ of original CAP area. (B) Effect of aglycemia duration on functional WM recovery. Averaged CAP areas are shown for three durations of aglycemia $(30,60$, or 90 $\min ; n=8$ ). There is a roughly linear decrease in WM recovery (i.e., CAP recovery) with increasing duration of aglycemia. (C) Under control conditions (i.e., $10 \mathrm{mM}$ bath glucose prior to aglycemia), glycogen was present and latency to CAP failure was $11.56+/-2.04 \mathrm{~min}(\mathrm{n}=8)$. After glycogen depletion (see text), latency to CAP failure was $4.33+/-2.34 \min \left(n=6 ; p<4.8 \times 10^{-5}\right.$ compared to latency in glycogen-containing MONs). Recovery of function after $60 \mathrm{~min}$ aglycemia, measured by CAP area, was significantly greater in glycogencontaining MONs (44.2 $\pm 4.9 \%$ vs. $21.8 \pm 4.5 \%$; $p=0.0016$; see text) (D) Age does not affect the vulnerability of WM to aglycemic dysfunction or injury. There were no significant differences in CAP recoveries after 60 min of aglycemia in animals of different ages (1, 3, 10 or 24-month-old). (Identical experiments done with 90 min aglycemia gave similar results (data not shown)). For clarity, SEM for CAPs was plotted every 3 min, not for every CAP.

\section{Figure 2.}

NMDA-type glutamate receptors (NMDARs) were involved in aglycemic WM injury. (A) Comparison of effects of NMDA-type and AMPA/kainate-type GluR antagonists (MK801 and NBQX, respectively) on WM recovery from 90 min of aglycemia. Application of MK801 $(15 \mu \mathrm{M})$ delayed CAP loss during aglycemia and dramatically improved CAP recovery (control: $5.0 \pm 1.2 \%, n=8$; MK801: 
$30.8 \pm 5.5 \%, n=9, p=0.0006)$. Application of NBQX $(30 \mu \mathrm{M})$ had no effect on the time course of CAP loss during aglycemia but produced a small improvement in CAP recovery $(12.2 \pm 1.8 \%, n=4, p=0.007$ compared to control). (B) Another NMDAR antagonist, 7-CKA (glycine binding site blocker) also greatly improved CAP recovery after 90 min of aglycemia (control: $6.4 \pm 2.6 \%, n=4 ; 7-C K A: 34.9$ $\pm 10.1 \%, n=5, p<0.001$ ). (C) Adding NBQX to MK801 did not improve CAP recovery after $90 \mathrm{~min}$ of aglycemia $(33.8+5.6 \%, \mathrm{n}=11)$ beyond what was seen with MK801 alone $(30.8 \pm 5.5 \%, n=9)$. MONs from three month-old animals were used in these experiments.

\section{Figure 3.}

Deleting bath $\mathrm{Mg}^{2+}$ or adding glycine worsened recovery after aglycemia. (A) Three separate 60 min aglycemia experiments are superimposed: control condition (' $2 \mathrm{mM} \mathrm{Mg}{ }^{2+}$ ), $\mathrm{Mg}^{2+-}$ free ACSF ('OmM Mg${ }^{2+}$ ') and $\mathrm{Mg}^{2+-}$ free ACSF with $15 \mu \mathrm{M}$ MK801 ('0mM Mg${ }^{2+}+\mathrm{MK} 801$ '). Superfusion conditions (i.e., $0 \mathrm{Mg}^{2+}+$ MK801) were maintained starting $30 \mathrm{~min}$ before, during, and $30 \mathrm{~min}$ after aglycemia ('0 $\mathrm{Mg}^{2+}$ ' bar). Reducing $\left[\mathrm{Mg}^{2+}\right]_{0}$ shortened the latency and accelerated the rate of decline of the CAP during aglycemia. Reducing $\left[\mathrm{Mg}^{2+}\right]_{0}$ also worsened aglycemic injury (control: $48.2 \pm 3.7 \%, \mathrm{n}=19$ vs. $0\left[\mathrm{Mg}^{2+}\right]_{0}: 14.9 \pm 4.4 \%, \mathrm{n}=7, \mathrm{p}$ $\left.=4.18 \times 10^{-5}\right)$. The effect of reducing $\left[\mathrm{Mg}^{2+}\right]_{0}$ was nullified in the presence of 15 $\mu \mathrm{M}$ MK801 (54.03 $\pm 10.6 \%, n=9, p>0.5$ compared to control). (B) Two separate 60 min aglycemia experiments are superimposed: control condition ("0 mM glycine") and $1 \mathrm{mM}$ glycine in ACSF. Addition of $1 \mathrm{mM}$ glycine significantly decreased CAP recovery from $60 \mathrm{~min}$ of aglycemia $(36.51 \pm 7.0 \%, n=8$ vs. 48.19 $\pm 1.7 \%, n=19 ; p<0.03)$. The presence of glycine also accelerated CAP decline during aglycemia. All experiments were done on three-month-old animals.

\section{Figure 4.}

Lactate in MON extracellular space decreased during aglycemia, associated with an alkaline shift in extracellular pH. (A) An enzyme electrode directly measured extracellular lactate concentration, [lactate-]o. The active sensor (dark gray) of the 
enzyme electrode was pressed alongside the optic nerve. Stimulus-evoked CAPs were simultaneously elicited and recorded via suction electrodes. (B) The [lactate-]o was zero in the bath (not shown) but averaged about $0.5 \mathrm{mM}$ at the edge of the optic nerve. After about 5 minutes of aglycemia, [lactate-]o fell steeply and reached nearly zero after about 10 to $15 \mathrm{~min}$. When glucose is reintroduced, [lactate-]o rapidly increases and briefly overshoots the original baseline level, before returning to a level that is very close to the original value. (C) Extracellular $\mathrm{pH}\left(\mathrm{pH}_{0}\right)$ within the nerve ( 7.2), measured with a $\mathrm{pH}$-sensitive microelectrode, was about $0.2 \mathrm{pH}$ unit lower than bath $\mathrm{pH}$ (7.4). During aglycemia, $\mathrm{pH}_{\circ}$ increased with a time course that mirrored the drop in [lactate-]o (a typical [lactate $]_{0}$ trace is superimposed for comparison). The $\mathrm{pH}_{\mathrm{o}}$ during aglycemia plateaued at $\sim 7.4$. When glucose was reintroduced, $\mathrm{pH}_{\circ}$ underwent an acid shift with a similar time course to the increase in [lactate-]o. A typical CAP trace during aglycemia is shown for comparison (see text). (D) Acidifying the ACSF (by lowering the concentration of $\mathrm{HCO}_{3}$ ) from $\mathrm{pH} 7.4$ to 7.0 improved CAP recovery after 90 min of aglycemia from $4.97 \pm 1.23 \%(n=8)$ to $13.38 \pm 2.9 \%(n=6 ; p=$ 0.012). The recovery segment of this experiment is expanded in the inset.

\section{Figure 5.}

Aglycemia-induced WM injury depended on extracellular $\mathrm{Ca}^{2+}$. MONs were exposed to aglycemia in normal ACSF (containing $2 \mathrm{mM} \mathrm{Ca}^{2+}$ ) or in ACSF with no $\mathrm{Ca}^{2+}\left(\mathrm{Mg}^{2+}\right.$ was adjusted to $4 \mathrm{mM}$ to maintain constant divalent cation concentration). The $\mathrm{Ca}^{2+}$-free condition was started $15 \mathrm{~min}$ before aglycemia and discontinued $15 \mathrm{~min}$ after aglycemia. Note that the $0 \mathrm{Ca}^{2+} \mathrm{ACSF}$ caused a small decrease in CAP area when introduced. (A) Perfusion with $\mathrm{Ca}^{2+}$-free ACSF led to nearly complete CAP recovery from 90 min aglycemia in 3-month-old MONs (control recovery: $4.97 \pm 1.2 \%, n=8$ vs. $\mathrm{Ca}^{2+}$-free: $86.93 \pm 3.6 \%, n=4$; $\mathrm{p}<0.00001$ ) and (B) in 10-month-old MONs (control: $6.86 \pm 2.06 \%, n=5$ vs. $\mathrm{Ca}^{2+}$-free: $\left.84.54 \pm 9.8 \%, \mathrm{n}=6 ; \mathrm{p}<0.00002\right)$.

\section{Figure 6.}


Summary of experimental results on the pathophysiology of aglycemia-induced WM injury. (A) Histograms showing CAP recovery from 90 min of aglycemia under different conditions (see text). The control ('con') level of recovery is shown to the left in each panel. (B) Histograms showing CAP recovery from 60 min of aglycemia under different conditions (see text). Statistical differences shown for comparison with control recovery ('con'). ${ }^{*}=p<0.05,{ }^{* *}=p<0.002,{ }^{* * *}=p<$ 0.0001. Error bars indicate SEM.

\section{Figure 7.}

Aglycemia reduced extracellular glutamate release but increased extracellular aspartate release. (A) Aglycemia for 40 min was not associated with glutamate release ('standard technique'; see methods). Subsequent oxygen-glucose deprivation (i.e., OGD), however, caused a large increase in normalized extracellular glutamate release after about $20 \mathrm{~min}$. (B) Aglycemia for 40 min was not associated with aspartate release ('standard technique'). Subsequent OGD, however, increased extracellular aspartate release after about $20 \mathrm{~min}$. (C) Using a modified technique that increased the resolution of amino acid release (see text), it can be seen that aglycemia reduced glutamate release. (D) Using the modified technique as in panel $\mathrm{C}$, aglycemia increased aspartate release. (E) The tissue (or intracellular) content of aspartate, glutamate and glycine is shown under control condition- and after $30 \mathrm{~min}$ of aglycemia. During aglycemia, the tissue content of aspartate increased about four-fold, the tissue content of glutamate fell about four-fold and the tissue content of glycine did not change significantly. ${ }^{* *}=p<0.001(\mathrm{~F})$ Application of the glutamate transporter inhibitor, TBOA (10mM), significantly improved CAP recovery after $90 \mathrm{~min}$ and $60 \mathrm{~min}$ aglycemia of recovery (control $=4.97 \pm 1.01 \%, n=8 ;$ TBOA $=\_20.23 \pm 4.55 \%, n=$ $7 ; p<0.005)$.

\section{Figure 8.}

Model comparing the mechanisms of hypoglycemic and ischemic WM injury. (A) WM under 'normal' conditions. In the presence of normal $\mathrm{O}_{2}$ and glucose, 
astrocytes have adequate ATP for the $\mathrm{Na}^{+}$pump to maintain a steep $\mathrm{Na}^{+}$ gradient that powers glutamate (also aspartate) uptake; consequently, [glutamate- $]_{\mathrm{o}}$ (and [aspartate-] $]_{\mathrm{o}}$ ) is low. The $\mathrm{pH}_{\mathrm{o}}$ is $\sim 7.2$. (B) WM during ischemia. Under ischemic conditions, energy metabolism in astrocytes persists until glycogen is exhausted (\#1). Acidosis accompanies ischemia and protons block ion fluxes in NMDA-type glutamate receptors (NMDARs), preventing them from participating in the injury cascade (\#2). The intracellular content of $\mathrm{Na}^{+}$ $\left(\left[\mathrm{Na}^{+}\right]_{1}\right)$ increases in the absence of ATP (\#3) causing glutamate release into the extracellular space via reverse $\mathrm{Na}^{+}$-dependent glutamate transport (probably from astrocytes, as shown)(\#4). In turn, glutamate activates AMPA/kainate receptors on oligodendrocytes and their processes (\#5), including myelin, leading to intracellular ionic derangements and irreversible injury (\#6; for details, see ${ }^{28}$ ) (C) WM during hypoglcemia. With severe hypoglycemia, or aglycemia, energy metabolism persists until glycogen and other substrates, like glutamate, are exhausted (\#1). In the absence of glucose, glutamate is consumed in the Krebs cycle as a short-term substrate and its intracellular concentration falls (\#2). The Krebs cycle now produces aspartate (from accumulating oxaloacetate) and the intracellular concentration of aspartate increases 4-fold (\#3; see text). As ATP falls, the $\mathrm{Na}^{+}$pump fails and $\left[\mathrm{Na}^{+}\right]_{i}$ increases leading to reversal of $\mathrm{Na}^{+}$ dependent glutamate/aspartate transport (\#4). Because intracellular glutamate is decreased, and aspartate is increased, it is aspartate that is transported into the extracellular space (\#4). The [lactate] falls, $\mathrm{pH}_{\mathrm{o}}$ increases and the 'proton' block of NMDARs is relieved (see text)(\#5). Aspartate is a high-affinity agonist for NMDA receptors (NMDARs) and activates these causing toxic ion fluxes, especially $\mathrm{Ca}^{2+}$ influx(\#6), and damage to oligodendrocytes and myelin (\#7).

\section{References:}

1. Agardh CD, Rosen I. Neurophysiological recovery after hypoglycemic coma in the rat: correlation with cerebral metabolism. J Cereb Blood Flow Metab. 1983 Mar;3(1):78-85. 
2. Arky RA. Hypoglycemia associated with liver disease and ethanol. Endocrinology and metabolism clinics of North America. 1989 Mar;18(1):75-90. 3. Davis EA, Jones TW. Hypoglycemia in children with diabetes: incidence, counterregulation and cognitive dysfunction. Journal of pediatric endocrinology \& metabolism : JPEM. 1998 Mar;11 Suppl 1:177-82.

4. Lincoln NB, Faleiro RM, Kelly C, Kirk BA, Jeffcoate WJ. Effect of long-term glycemic control on cognitive function. Diabetes care. 1996 Jun;19(6):656-8.

5. Auer RN. Hypoglycemic brain damage. Metab Brain Dis. 2004 Dec;19(34):169-75.

6. Brown AM, Wender R, Ransom BR. Ionic mechanisms of aglycemic axon injury in mammalian central white matter. $J$ Cereb Blood Flow Metab. 2001;21(4):385-95.

7. Warren RE, Frier BM. Hypoglycaemia and cognitive function. Diabetes, obesity \& metabolism. 2005 Sep;7(5):493-503.

8. McCrimmon RJ, Ryan CM, Frier BM. Diabetes and cognitive dysfunction. Lancet. 2012 Jun 16;379(9833):2291-9.

9. $\mathrm{Ma} \mathrm{JH}, \mathrm{Kim} \mathrm{YJ}$, Yoo WJ, et al. MR imaging of hypoglycemic encephalopathy: lesion distribution and prognosis prediction by diffusionweighted imaging. Neuroradiology. 2009 Oct;51(10):641-9.

10. Johkura K, Nakae Y, Kudo Y, Yoshida TN, Kuroiwa Y. Early diffusion MR imaging findings and short-term outcome in comatose patients with hypoglycemia. AJNR Am J Neuroradiol. 2012 May;33(5):904-9.

11. Ransom BR, Baltan SB. Axons get excited to death. Ann Neurol. 2009 Feb;65(2):120-1.

12. Matute C, Domercq M, Perez-Samartin A, Ransom BR. Protecting White Matter From Stroke Injury. Stroke. 2012 Dec 4.

13. Stys PK, Ransom BR, Waxman SG. Compound action potential of nerve recorded by suction electrode: a theoretical and experimental analysis. Brain Res. 1991;546(1):18-32.

14. Li S, Stys PK. Mechanisms of ionotropic glutamate receptor-mediated excitotoxicity in isolated spinal cord white matter. J Neurosci. 2000;20(3):1190-8.

15. Peng W, Cotrina ML, Han X, et al. Systemic administration of an antagonist of the ATP-sensitive receptor $\mathrm{P} 2 \mathrm{X} 7$ improves recovery after spinal cord injury. Proc Natl Acad Sci U S A. 2009 Jul 28;106(30):12489-93.

16. Fern R, Moller T. Rapid ischemic cell death in immature oligodendrocytes: a fatal glutamate release feedback loop. J Neurosci. 2000 Jan 1;20(1):34-42. 17. Brown AM, Westenbroek RE, Catterall WA, Ransom BR. Axonal L-type $\mathrm{Ca} 2+$ channels and anoxic injury in rat CNS white matter. J Neurophysiol. 2001 Feb;85(2):900-11.

18. Baltan S, Besancon EF, Mbow B, Ye Z, Hamner MA, Ransom BR. White matter vulnerability to ischemic injury increases with age because of enhanced excitotoxicity. J Neurosci. 2008 Feb 6;28(6):1479-89.

19. McDonald JW, Althomsons SP, Hyrc KL, Choi DW, Goldberg MP. Oligodendrocytes from forebrain are highly vulnerable to AMPA/kainate receptormediated excitotoxicity. Nat Med. 1998 Mar;4(3):291-7. 
20. Sanchez-Gomez MV, Matute C. AMPA and kainate receptors each mediate excitotoxicity in oligodendroglial cultures. Neurobiol Dis. 1999 Dec;6(6):475-85.

21. Alberdi E, Sanchez-Gomez MV, Marino A, Matute C. $\mathrm{Ca}(2+)$ influx through AMPA or kainate receptors alone is sufficient to initiate excitotoxicity in cultured oligodendrocytes. Neurobiol Dis. 2002 Mar;9(2):234-43.

22. Tekkok SB, Goldberg MP. AMPA/Kainate receptor activation mediates hypoxic oligodendrocyte death and axonal injury in cerebral white matter. $J$ Neurosci. 2001 Jun 15;21(12):4237-48.

23. Bakiri Y, Hamilton NB, Karadottir R, Attwell D. Testing NMDA receptor block as a therapeutic strategy for reducing ischaemic damage to CNS white matter. Glia. 2008 Jan 15;56(2):233-40.

24. Micu I, Jiang Q, Coderre E, et al. NMDA receptors mediate calcium accumulation in myelin during chemical ischaemia. Nature. 2006 Feb 23;439(7079):988-92.

25. Li S, Mealing GA, Morley P, Stys PK. Novel injury mechanism in anoxia and trauma of spinal cord white matter: glutamate release via reverse $\mathrm{Na}(+)-$ dependent glutamate transport. J Neurosci. 1999;19(14):RC16.

26. Hamner MA, Moller T, Ransom BR. Anaerobic function of CNS white matter declines with age. Journal of cerebral blood flow and metabolism : official journal of the International Society of Cerebral Blood Flow and Metabolism. 2011 Apr;31(4):996-1002.

27. Borrelli MJ, Carlini WG, Dewey WC, Ransom BR. A simple method for making ion-selective microelectrodes suitable for intracellular recording in vertebrate cells. J Neurosci Methods. 1985;15(2):141-54.

28. Tekkok SB, Ye Z, Ransom BR. Excitotoxic mechanisms of ischemic injury in myelinated white matter. J Cereb Blood Flow Metab. 2007 Sep;27(9):1540-52. 29. Ye ZC, Ransom BR, Sontheimer H. (1R,3S)-1-Aminocyclopentane-1,3dicarboxylic acid (RS-ACPD) reduces intracellular glutamate levels in astrocytes. J Neurochem. 2001 Nov;79(4):756-66.

30. Wender R, Brown AM, Fern R, Swanson RA, Farrell K, Ransom BR. Astrocytic glycogen influences axon function and survival during glucose deprivation in central white matter. J Neurosci. 2000;20(18):6804-10.

31. Brown AM, Tekkok SB, Ransom BR. Glycogen regulation and functional role in mouse white matter. J Physiol. 2003 Jun 1;549(Pt 2):501-12.

32. Underhill SM, Goldberg MP. Hypoxic injury of isolated axons is independent of ionotropic glutamate receptors. Neurobiol Dis. 2007 Feb;25(2):284-90.

33. Mathiesen $\mathrm{C}$, Varming $\mathrm{T}$, Jensen $\mathrm{LH}$. In vivo and in vitro evaluation of AMPA receptor antagonists in rat hippocampal neurones and cultured mouse cortical neurones. Eur J Pharmacol. 1998 Jul 24;353(2-3):159-67.

34. Traynelis SF, Wollmuth LP, McBain CJ, et al. Glutamate receptor ion channels: structure, regulation, and function. Pharmacological reviews. 2010 Sep;62(3):405-96. 
35. Brown AM, Evans RD, Black J, Ransom BR. Schwann cell glycogen selectively supports myelinated axon function. Ann Neurol. 2012 Sep;72(3):40618.

36. Lee J, Taira T, Pihlaja P, Ransom BR, Kaila K. Effects of $\mathrm{CO}_{2}$ on excitatory transmission apparently caused by changes in intracellular $\mathrm{pH}$ in the rat hippocampal slice. Brain Res. 1996;706(2):210-6.

37. Traynelis SF, Cull-Candy SG. Proton inhibition of N-methyl-D-aspartate receptors in cerebellar neurons. Nature. 1990;345(6273):347-50.

38. McCrimmon RJ, Frier BM. Hypoglycaemia, the most feared complication of insulin therapy. Diabete Metab. 1994 Nov-Dec;20(6):503-12.

39. Zhang K, Sejnowski TJ. A universal scaling law between gray matter and white matter of cerebral cortex. Proc Natl Acad Sci U S A. 2000 May 9;97(10):5621-6.

40. Ransom BR, Goldberg MP, Baltan SB. Molecular pathophysiology of white matter anoxic-ischemic injury. In: Mohr JP, editor. Stroke: pathophysiology, diagnosis and management. 5th edition ed. Philadelphia, PA: Elsevier/Saunders; 2011. p. 122-37.

41. Hansen AJ. Effect of anoxia on ion distribution in the brain. Physiol Rev. 1985;65(1):101-48.

42. Brown AM, Wender R, Ransom BR. Metabolic substrates other than glucose support axon function in central white matter. J Neurosci Res. 2001 Dec $1 ; 66(5): 839-43$.

43. Agardh CD, Folbergrova J, Siesjo BK. Cerebral metabolic changes in profound, insulin-induced hypoglycemia, and in the recovery period following glucose administration. J Neurochem. 1978 Nov;31(5):1135-42.

44. Pelligrino D, Siesjo BK. Regulation of extra- and intracellular $\mathrm{pH}$ in the brain in severe hypoglycemia. Journal of cerebral blood flow and metabolism : official journal of the International Society of Cerebral Blood Flow and Metabolism. 1981;1(1):85-96.

45. Sandberg $M$, Butcher SP, Hagberg $\mathrm{H}$. Extracellular overflow of neuroactive amino acids during severe insulin-induced hypoglycemia: in vivo dialysis of the rat hippocampus. J Neurochem. $1986 \mathrm{Jul} ; 47(1): 178-84$.

46. Salter MG, Fern R. NMDA receptors are expressed in developing oligodendrocyte processes and mediate injury. Nature. $2005 \mathrm{Dec}$ 22;438(7071):1167-71.

47. Rothman SM, Olney JW. Glutamate and the pathophysiology of hypoxic-ischemic brain damage. Ann Neurol. 1986;19(2):105-11.

48. Stys PK, Waxman SG, Ransom BR. Na(+)-Ca2+ exchanger mediates $\mathrm{Ca} 2+$ influx during anoxia in mammalian central nervous system white matter. Ann Neurol. 1991;30(3):375-80.

49. Suh SW, Hamby AM, Swanson RA. Hypoglycemia, brain energetics, and hypoglycemic neuronal death. Glia. 2007 Sep;55(12):1280-6.

50. Nellgard B, Wieloch T. Cerebral protection by AMPA- and NMDA-receptor antagonists administered after severe insulin-induced hypoglycemia. Exp Brain Res. 1992;92(2):259-66. 
